\title{
Altered melatonin secretion and circadian gene expression with increased proinflammatory cytokine expression in early-stage sepsis patients
}

\author{
CAI-XIA LI ${ }^{1}$, DONG-DONG LIANG ${ }^{2}$, GUO-HAO XIE ${ }^{1}$, BAO-LI CHENG ${ }^{1}$, QI-XING CHEN ${ }^{1}$, \\ SHUI-JING WU ${ }^{1}$, JUN-LU WANG ${ }^{2}$, WILLIAM $\mathrm{CHO}^{3}$ and XIANG-MING FANG ${ }^{1}$
}

${ }^{1}$ Department of Anesthesiology, The First Affiliated Hospital, School of Medicine, Zhejiang University, Hangzhou;

${ }^{2}$ Department of Anesthesiology, The First Affiliated Hospital of Wenzhou Medical College, Wenzhou;

${ }^{3}$ Department of Clinical Oncology, Queen Elizabeth Hospital, Hong Kong, P.R. China

Received October 10, 2012; Accepted January 14, 2013

DOI: $10.3892 / \mathrm{mmr} .2013 .1331$

\begin{abstract}
Inflammatory and immune responses, as well as melatonin secretion, are affected by circadian regulation. Abnormal circadian rhythm of melatonin release has been reported to be associated with the later stages of sepsis; however, its role in the early stages of sepsis is unclear. We studied 11 septic and 11 non-septic patients in our intensive care unit (ICU). Peripheral blood was drawn at 4-h intervals on the first day, beginning at 2:00 p.m., over a total period of $24 \mathrm{~h}$. Plasma levels of melatonin, tumor necrosis factor $\alpha$ (TNF- $\alpha$ ) and interleukin 6 (IL-6) were measured by radioimmunoassay or enzyme-linked immunosorbent assay (ELISA). Messenger RNA levels of circadian genes Cry-1 and Per-2 were analyzed using quantitative real-time PCR. Results show the circadian rhythm of melatonin secretion was altered in the early stages of sepsis. The melatonin secretion acrophase occurred earlier in septic patients at 6:00 p.m., compared with at 2:00 a.m. in non-septic ICU patients. Compared with the non-septic group, both $C r y-1$ and $P e r-2$ expression were significantly decreased while TNF- $\alpha$ and IL-6 expression were significantly increased in septic patients [TNF- $\alpha, 64.1$ (43.6-89.1) vs. 11.4 (10.4-12.5) ng/ml; IL-6, 41.2 (35.7-50.8) vs. 19.1 (16-136.7) ng/ml; median (range), both $\mathrm{P}=0.04]$. The peak concentrations of TNF- $\alpha$ and IL- 6 were shown to be in concordance with the rhythm of melatonin secretion. The circadian rhythm of melatonin secretion and circadian gene expression
\end{abstract}

Correspondence to: Professor Xiang-Ming Fang, Department of Anesthesiology, The First Affiliated Hospital, School of Medicine, Zhejiang University, No. 79 Qingchun Road, Hangzhou, Zhejiang, P.R. China

E-mail: xiangming_fang@163.com

Professor Jun-Lu Wang, Department of Anesthesiology, The First Affiliated Hospital of Wenzhou Medical College, No. 2 Fuxue Lane, Wenzhou, Zhejiang, P.R. China

E-mail: wangjunluxuesheng@yahoo.com.cn

Key words: melatonin secretion, sepsis, TNF- $\alpha$, gene expression were altered in the early stages of sepsis, which likely led to the changes in pro-inflammatory cytokine release. These findings shed light on the potential link between circadian rhythm and the progression of early-stage sepsis.

\section{Introduction}

Despite advances in diagnostics and intensive care treatment, morbidity and mortality remain considerably high in septic patients (1,2). A dysregulated systemic inflammatory response is one of the main characteristics associated with sepsis (3). Previous studies attempted to inhibit inflammatory pathways via various methods, including ciglitazone, lipid-rich nutrition and C1-esterase inhibitors. However, none of these drugs have been shown to effectively reduce the mortality rate (4-6). Therefore, the discovery of novel biomarkers and therapeutic targets involved in the pathophysiology of sepsis is in high demand by patients and caregivers.

Melatonin, synthesized by the pineal gland, is a key molecule of the central 'master' clock, which controls circadian rhythmicity (7). The plasma melatonin level has been shown to vary according to the circadian cycle. In healthy humans, melatonin secretion occurs almost exclusively during night hours starting between 9:00 p.m. and 11:00 p.m., reaching peak serum levels between 1:00 a.m. and 3:00 a.m., and then falling to low baseline values between 7:00 a.m. and 9:00 a.m. (8). Previous studies have shown that melatonin regulates immune function and inflammatory response, as well as possesses indirect antioxidant activity $(9,10)$. In intensive care unit (ICU) patients, the normal cyclic change between daytime and sleep during the night is disturbed, particularly if continuous application of ataractic and other drugs is required. A previous clinical study has also demonstrated that circadian rhythm is disrupted in sedated critically ill patients with severe sepsis (11). Melatonin may ameliorate the sleep pattern of these patients and improve the survival rate (12).

A number of genes, such as Clock, Cry 1-2, Perl-2, Rev-erb and Bmall, have been shown to participate in the regulation of circadian rhythmicity (13). Oscillations of circadian genes have been identified in many peripheral tissues $(14,15)$. 
These circadian genes may be meticulously adjusted by a master clock, which is located in the suprachiasmatic nucleus within the brain hypothalamus. The mechanisms by which the 'master' clock and the peripheral clocks interact are not completely understood.

A recent clinical trial demonstrated that endotoxin did not affect the melatonin secretion rhythm, but suppressed the expression of peripheral circadian genes in healthy volunteers (16). Previous studies have also shown that the circadian activity of the clocks is modified during periods of systemic inflammation. Tumor necrosis factor $\alpha(\mathrm{TNF}-\alpha)$ and interleukin 6 (IL-6), two prominent pro-inflammatory cytokines produced during the acute phase of sepsis, have also been implicated in the regulation of circadian activity $(15,17)$. It has also been shown that the normal periodicity of urinary 6-sulfatoxymelatonin $\alpha$ ( $\alpha$ MT6s) excretion (a metabolic product of melatonin) was eliminated and phase amplitudes were much lower in the late stages of severe septic patients compared to non-septic patients (11). Thus, it is possible that melatonin rhythm is also altered in the early stages of sepsis.

In the current study, we investigated the altered circadian rhythm of melatonin and expression of two circadian genes in peripheral blood leukocytes (PBLs) of septic patients during the first $24 \mathrm{~h}$ of ICU treatment. Additionally, we assessed the release of two pro-inflammatory cytokines, TNF- $\alpha$ and IL-6, in the serum of septic and non-septic patients.

\section{Materials and methods}

Ethics statement. Written informed consent forms were signed by all patients or, in case of unconsciousness, by their close relatives. The study was approved by the ethical committee of the First Affiliated Hospital of Wenzhou Medical College, Wenzhou, China.

Patients. Two groups of ICU patients were enrolled in this study: the septic group was comprised of 11 patients who were diagnosed with sepsis, and the control group consisted of 11 non-septic patients. All patients in the present study were admitted to the First Affliated Hospital of Wenzhou Medical College ICU and subjected to a similar environment, including the light-dark cycle and the same diet. Sepsis was defined as the systemic inflammatory response syndrome (SIRS) resulting from infection according to the American College of Chest Physicians and the Society of Critical Care Medicine Consensus Conference (18). SIRS was manifested by two or more of the following conditions as a result of infection: temperature $>38.3^{\circ} \mathrm{C}$ or $<35.6^{\circ} \mathrm{C}$; heart rate $>90$ beats $/ \mathrm{min}$; tachypnea $>20 /$ min, or hypocapnia $\left(\mathrm{PaCO}_{2}<32 \mathrm{mmHg}\right)$; WBC $>12,000$ cells/ $\mathrm{mm}^{3}$ or $<4,000$ cells $/ \mathrm{mm}^{3}$, or $>10 \%$ immature (band) forms (19). In the septic group, four of the 11 septic patients succumbed to disease during their ICU stay, whereas seven patients recovered from sepsis (Table I). Patients with insomnia, mental illness, those engaged in shift work prior to admission to the hospital, or patients receiving $\beta$-blockers, melatonin and blocking agents of melatonin were excluded from the study.

Study course. Peripheral blood samples were collected in evacuated EDTA glass tubes and Paxgene tubes (Qiagen, Valencia, CA, USA) at 2:00 p.m. on the day of admission to the
ICU and every $4 \mathrm{~h}$ thereafter, up to $24 \mathrm{~h}$, through an indwelling arterial line. Plasma was obtained after blood samples were centrifuged at $1,400 \mathrm{x} \mathrm{g}$ for $5 \mathrm{~min}$ and was stored at $-80^{\circ} \mathrm{C}$ for further analysis. The blood in the Paxgene tubes was used for total RNA isolation.

Laboratory analyses. Total RNA was isolated from the peripheral blood collected in the Paxgene tubes using PAXgene Blood RNA kit (Qiagen), according to the manufacturer's instructions. Briefly, RNA was quantified using an Agilent 2100 Bioanalyzer (Agilent Technologies, Palo Alto, CA, USA). cDNA was synthesized using GoScript ${ }^{\mathrm{TM}}$ Reverse Transcription System (Promega, Beijing, China). Real-time relative quantitative RT-PCR was performed to detect mRNA expression levels of the target genes Cry-1 and Per-2, which are the key genes in the regulation of circadian clock function, as well as the reference gene $\beta$-actin, using the 7500 Real-Time PCR System (Applied Biosystems, Foster City, CA, USA). The PCR system contained 1X TaqMan ${ }^{\circledR}$ Gene Expression Master Mix (Applied Biosystems), $250 \mathrm{nM}$ each of the forward and reverse primers and $0.15 \mu \mathrm{M}$ hybridization probe in a volume of $20 \mathrm{ml}$. The sequence of the primers and probes were as follows: Cry-1: forward primer 5'-GCA TTT ATG CTC CAA TCT GCA TC-3', reverse primer 5'-AGG AGG GTT GGA TTC ATC ATC TAG-3', probe 5'-CAA ATA CCT TCA TTC CTT C-3'; Per-2: forward primer 5'-CCT CAG GAG CGT GAA GCA G-3', reverse primer 5'-TCA CAA TGT GCT CAG AGG TAA CG-3', probe 5'-TGA AAG CCA ATG AAG AGT-3'; $\beta$-actin: forward primer 5'-CAT TGC CGA CAG GAT GCA-3', reverse primer 5'-CAT CTG CTG GAA GGT GGA CAG-3', probe 5'-AGC AAT GAT CTT GAT CTT CA-3'. The PCR cycling parameters were set as follows: 1 cycle at $95^{\circ} \mathrm{C}$ for $3 \mathrm{~min}, 45$ cycles at $95^{\circ} \mathrm{C}$ for $30 \mathrm{sec}$ and $55^{\circ} \mathrm{C}$ for $30 \mathrm{sec}$ and 1 cycle at $95^{\circ} \mathrm{C}$ for $1 \mathrm{~min}$, followed by a melting curve initiating at $55^{\circ} \mathrm{C}$ and increasing in $0.5^{\circ} \mathrm{C}$ increments for 80 steps. For every sample, target genes and reference genes were amplified simultaneously in separate triplicate wells. Relative gene expression analysis was performed using the $2^{-\Delta \Delta \mathrm{Ct}}$ method. Each sample was normalized with the loading control $\beta$-actin. The $\mathrm{Ct}$ values were obtained from the means of the triplicate experiments. Data are expressed as fold change relative to time at 2:00 p.m.

Melatonin concentrations in plasma were determined using a direct melatonin radioimmunoassay kit (Rocky Mountain Diagnostics, Colorado Springs, CO, USA), according to the manufacturer's instructions.

Plasma TNF- $\alpha$ and IL- 6 levels were measured using a Human TNF- $\alpha$ Quantikine enzyme-linked immunosorbent assay (ELISA) kit and a Human IL-6 Quantikine ELISA kit, respectively (R\&D systems, Minneapolis, MN, USA), according to the manufacturer's instructions.

Statistical analysis. Statistical analysis was performed using SPSS 17.0 (SPSS, Inc., Chicago, IL, USA). Continuous variables are reported as the means \pm SD or the median with interquartile range (IQR). Categorical variables were reported as frequencies and percentages. Baseline data between septic and non-septic patients were compared using the Student's t-test or Chi-square test. Messenger RNA expression levels of circadian genes are presented as the means \pm SD, and Student's t-test was applied for the comparisons. Comparisons between the two groups 
Table I. Diagnosis of participants $(n=22)$.

\begin{tabular}{lr}
\hline Diagnosis & Patient no. \\
\hline Septic group & 2 \\
Septic shock & 2 \\
Acute obstructive suppurative cholangitis & 2 \\
Multiple trauma & 2 \\
Soft tissue infection & 1 \\
Acute gangrenous appendicitis & 1 \\
Intestinal perforation & 1 \\
Acute pyelonephritis & 11 \\
Total & \\
Non-septic group & 3 \\
Cerebral hemorrhage & 2 \\
Liver and kidney failure & 1 \\
Acute poisoning & 2 \\
Craniocerebral injury & 1 \\
Coronary heart disease & 1 \\
Heat stroke & 1 \\
Status asthmaticus & 11 \\
Total &
\end{tabular}

were performed with the Mann-Whitney $\mathrm{U}$ test. $\mathrm{P}<0.05$ was considered to indicate a statistically significant difference.

\section{Results}

Patient characteristics. A total of 22 ICU patients (11 with sepsis and 11 without sepsis) were recruited for this study. Patient characteristics at the time of enrollment are listed in Tables I and II. Baseline data between the two groups were comparable, with the exception of a decreased mean arterial pressure (MAP) and a higher sepsis-related organ failure assessment in patients with sepsis (Table II).

Secretion of melatonin. Melatonin secretion was monitored at 4-h intervals over $24 \mathrm{~h}$ in each patient. Plasma levels of melatonin in septic and non-septic patients within the study period were $39.52(25.81,53.49) \mathrm{pg} / \mathrm{ml}$ (median with IQR) and 17.04 $(7.06,32.31) \mathrm{pg} / \mathrm{ml}$, respectively. Although melatonin concentrations tended to be higher in the septic group, statistical significance was not observed $(\mathrm{P}=0.055)$. Notably, the peak time for melatonin secretion in sepsis patients ( 6:00 p.m.) was clearly distinct from non-sepsis patients ( 2:00 a.m.). Serum melatonin levels were significantly increased at 2 p.m. [20.97 (7.91, 80.28) pg/ml and 6:00 p.m. (21.62 (14.10, 113.95) pg/ml] in septic patients when compared with that in the control group $[0.4(0,10.09) \mathrm{pg} / \mathrm{ml}$ at 2:00 p.m. and $0(0,18.04) \mathrm{pg} / \mathrm{ml}$ at 6:00 p.m.; $\mathrm{P}=0.03$ for both time points (Fig. 1)].

Circadian gene expression. The circadian genes Cry-1 and Per-2 were detected at the mRNA level in all the patients studied. mRNA levels of the target genes are relative to those of the housekeeping gene $\beta$-actin The expression of $C r y-1$ and Per-2 was significantly decreased from 2:00 p.m. to 10:00 a.m.

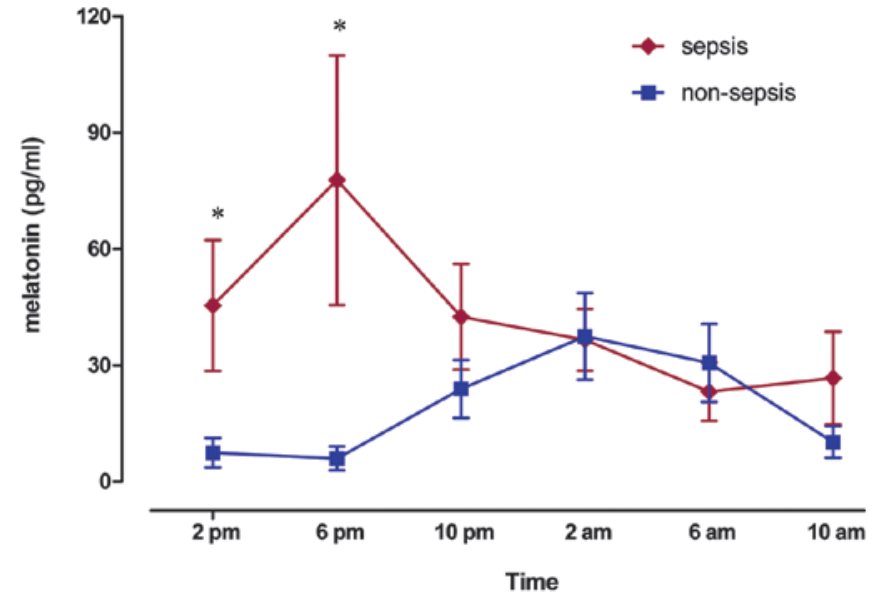

Figure 1. Secretary rhythm of melatonin in critically ill patients $(n=22)$ Plasma melatonin concentrations were determined in peripheral blood from septic $(n=11)$ and non-septic patients $(n=11)$ at the indicated time points. ${ }^{*} \mathrm{P}<0.05$ compared with non-septic patients.
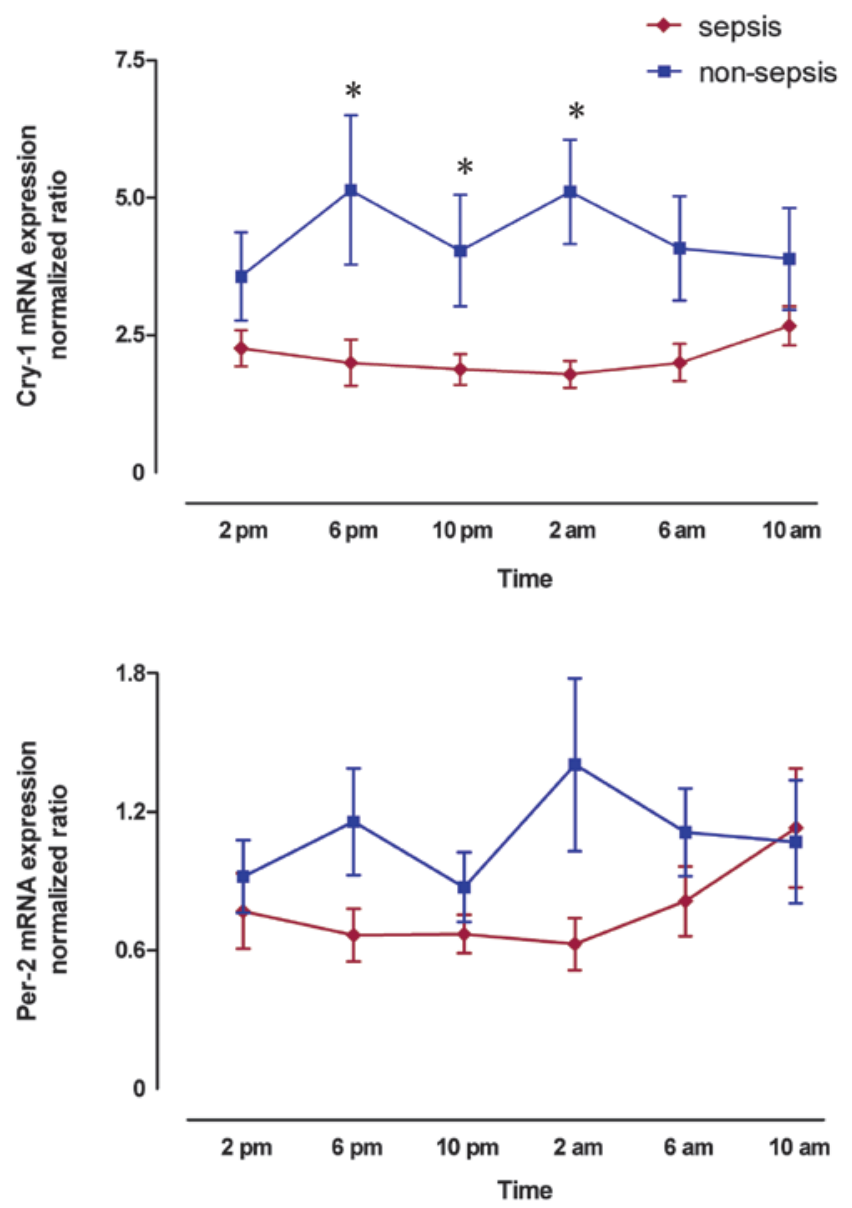

Figure 2. mRNA expression of circadian genes Per-2 and Cry-1 in critically ill patients. Expression of Cry-1 and Per-2 in peripheral blood from septic $(\mathrm{n}=11)$ and non-septic patients $(\mathrm{n}=11)$ at the indicated time points. mRNA levels were analyzed by real-time PCR. Cry-1 and Per-2 expression was decreased significantly in the peripheral blood of septic patients. ${ }^{*} \mathrm{P}<0.05$ compared with sepsis patients.

in septic patients compared with the non-septic patients (2.10 \pm 0.32 vs. $4.30 \pm 0.66, \mathrm{p}<0.001$ for $C r y-1$ and $0.78 \pm 0.12$ vs. $1.09 \pm 0.19, \mathrm{P}=0.01$ for Per-2; Fig. 2). 
Table II. Comparison of baseline data between septic and non-septic ICU patients.

\begin{tabular}{|c|c|c|c|}
\hline Parameter & $\begin{array}{l}\text { Septic patients } \\
\qquad(\mathrm{n}=11)\end{array}$ & $\begin{array}{l}\text { Non-septic patients } \\
\qquad(\mathrm{n}=11)\end{array}$ & P-value \\
\hline Age, years & $59.45 \pm 19.16$ & $62.55 \pm 15.24$ & NS \\
\hline Gender, male/female & $6 / 5$ & $2 / 9$ & NS \\
\hline Heart rate, bpm & $111.91 \pm 26.42$ & $99.91 \pm 28.16$ & NS \\
\hline MAP, $\mathrm{mmHg}$ & $71.82 \pm 24.96$ & $109.27 \pm 30.96$ & 0.006 \\
\hline APACHE score & $26.09 \pm 9.57$ & $21.55 \pm 5.46$ & NS \\
\hline SOFA score & $10.8 \pm 6.13$ & $6.45 \pm 2.84$ & 0.045 \\
\hline ICU stay, days & $7.82 \pm 3.34$ & $10.0 \pm 8.93$ & NS \\
\hline Hospital stay, days & $30.73 \pm 33.20$ & $24.82 \pm 20.05$ & NS \\
\hline Mechanical ventilation, number of patients & 6 & 9 & NS \\
\hline Vasoactive drug, number of patients & 6 & 6 & NS \\
\hline Prognosis, improvement/mortality & $7 / 4$ & $6 / 5$ & NS \\
\hline
\end{tabular}

MAP, mean arterial pressure; APACHE, acute physiology and chronic health evaluation; SOFA, sepsis-related organ failure assessment; NS, no significant difference; ICU, intensive care unit.
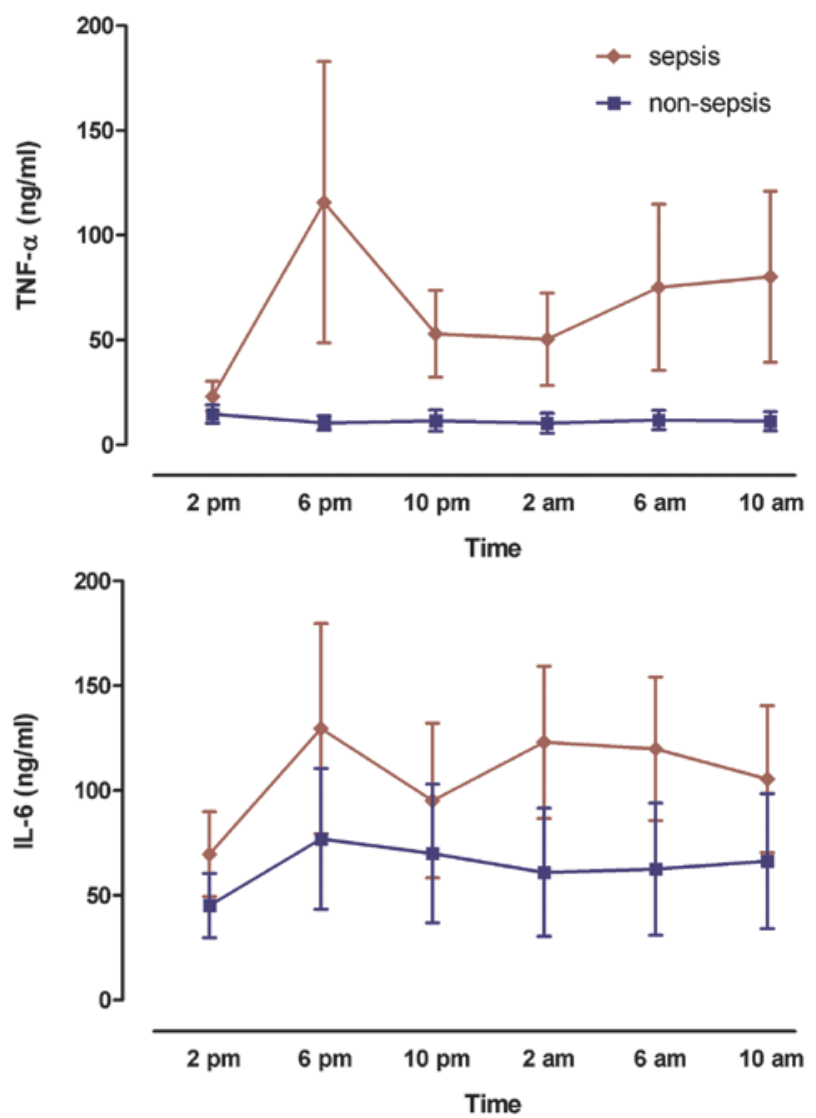

Figure 3. Plasma TNF- $\alpha$ and IL-6 concentrations in critically ill patients. Plasma TNF- $\alpha$ and IL- 6 concentrations in septic $(n=11)$ and non-septic patients $(\mathrm{n}=11)$ at indicated time points were determined by enzyme-linked immunosorbent assay.

Levels of plasma pro-inflammatory cytokines. We also monitored plasma TNF- $\alpha$ and IL-6 concentrations in both septic and non-septic patients at 4 -h intervals over $24 \mathrm{~h}$. TNF- $\alpha$ and IL-6 levels were significantly increased in septic patients [64.1 (43.6-89.126) and $41.2(35.7-50.8) \mathrm{ng} / \mathrm{ml}$, respectively] as compared with non-septic patients [11.4 (10.4-12.5) and 19.1 (16-136.7) ng/ml, respectively; $\mathrm{P}=0.04$ for both]. The peak concentrations of TNF- $\alpha$ and IL-6 occurred at 6:00 p.m. (Fig. 3), which was coincident with the rhythm of melatonin secretion. By contrast, no significant peak of TNF- $\alpha$ or IL-6 was observed in non-sepsis patients.

\section{Discussion}

The present study revealed altered circadian rhythm of melatonin secretion in patients with early sepsis, featuring a markedly shifted acrophase. The expression of clock genes Per-2 and Cry- 1 was significantly decreased in septic patients. By contrast, plasma levels of the major pro-inflammatory cytokines TNF- $\alpha$ and IL-6 increased in septic patients, reaching a peak at 6:00 p.m., which was consistent with the altered rhythm of melatonin secretion.

Altered sleep-wake rhythm, abnormal oxidative stress and use of drugs may affect the secretion of melatonin. Impaired melatonin secretion has been demonstrated in critically ill patients, including patients with late stage sepsis $(12,20)$. Mundigler et al demonstrated that the secretion of melatonin completely eradicated the natural rhythm of urinary $\alpha$ MT6 excretion in septic patients who had been in the ICU for at least one week (11). Considering the immunomodulatory and antioxidative role of melatonin during sepsis, abnormalities of melatonin secretion at the late stage of sepsis may be more noticeable. However, in the current study, the circadian rhythms of melatonin secretion also occurred in the early stage of sepsis, although its pattern was different. This finding is consistent with a previous study showing that the melatonin rhythms are intact in healthy individuals challenged by endotoxin, a model of very early-stage sepsis (16). These results suggest that abnormalities in melatonin rhythms in early and late sepsis may be induced by separate mechanisms.

Ten circadian clock genes have been identified in peripheral tissues, including Per-1-3, Cry-1 and Cry-2, Clock 
and Bmall, which coordinate with the master clock (19). Investigations in mice with genetic alterations in circadian genes revealed an elaborate relationship between the circadian clock and the immune inflammatory response (21). Downregulated circadian gene expression was reported in endotoxin-challenged rats (22) as well as endotoxin-treated healthy volunteers (16). Consistent with these observations, this study demonstrated that the expression of circadian genes (Per-2 and Cry-1) were significantly suppressed in PBLs of septic patients. Notably, this alteration appears to be independent of melatonin rhythm. Previous studies have demonstrated that several of the ten clock genes appeared to follow a similar rhythm in immune cells $(22,23)$. Godin and Buchman proposed that a systemic inflammatory response may cause uncoupling between day and night (24). We also showed that the suppression of peripheral circadian genes was independent of the melatonin rhythm, which provides some clues that there may be a severe misalignment between the central master clock and peripheral circadian clock genes in PBLs of septic patients.

Alteration of melatonin circadian rhythm is a result of multiple factors (20). Certain factors occur in serum during acute systemic inflammation and interact with the master clock; while others, such as exposure to certain environments, sedative drugs, endogenic catecholamines and even specific critical illness, may also interfere with the circadian rhythm (20). We observed that plasma TNF- $\alpha$ and IL-6 levels were significantly increased in septic patients, and peaked at 6:00 p.m., which is the same as the shifted rhythm peak of melatonin. Melatonin exerts many regulatory functions by modulating cellular behavior via binding to specific receptors and intracellular targets $(25,26)$. It is well known that melatonin, as an anti-inflammatory molecule, reduces oxidative tissue injuries by its antioxidant properties and selectively inhibits the late/chronic phases of inflammatory responses $(27,28)$. However, the current study cannot distinguish whether the elevated levels and altered circadian rhythms of pro-inflammatory cytokines TNF- $\alpha$ and IL- 6 are the result or the cause of melatonin alternation. Further studies are required to investigate the interaction between melatonin and proinflammatory cytokines such as TNF- $\alpha$ and IL-6.

In conclusion, the present study demonstrated that the circadian rhythm of melatonin secretion was altered in early septic patients, with a markedly shifted peak value. The pro-inflammatory cytokines TNF- $\alpha$ and IL- 6 were produced in a similar pattern as melatonin in patients with sepsis, while the expression of circadian genes (Cry-1 and Per-2) in PBLs was eliminated. The findings of the current study provide a novel correlation between melatonin secretion and pro-inflammatory cytokine release in sepsis. Considering its regulatory role in the immune response, melatonin may be a therapeutic target for sepsis. However, in this pilot study, it cannot be inferred from the current data whether the elevated levels and altered circadian rhythms of pro-inflammatory cytokines TNF- $\alpha$ and IL- 6 are simply caused by melatonin or if they represent a physiological counteracting mechanism during the early stage of sepsis. Further study is warranted to elucidate the mechanisms of altered circadian rhythm in sepsis.

\section{References}

1. Mackenzie I and Lever A: Management of sepsis. Br Med J 335: 929-932, 2007.

2. Dellinger RP, Levy MM, Carlet JM, Bion J and Parker MM Surviving Sepsis Campaign: international guidelines for management of severe sepsis and septic shock. Crit Care Med 36: 296-327, 2008

3. Riedemann NC, Guo RF and Ward PA: The enigma of sepsis. J Clin Invest 112: 460-467, 2003.

4. Chima RS, Hake PW, Piraino G, Mangeshkar P and Denenberg A: Ciglitazone ameliorates lung inflammation by modulating the inhibitor $\kappa \mathrm{B}$ protein kinase/nuclear factor $\kappa \mathrm{B}$ pathway after hemorrhagic shock. Crit Care Med 36: 2849-2857, 2008.

5. Lubbers T, De Haan JJ, Hadfoune M, Zhang Y and Luyer MD: Lipid-enriched enteral nutrition controls the inflammatory response in murine Gram-negative sepsis. Crit Care Med 38: 1996-2002, 2010

6. Dorresteijn MJ, Visser T, Cox LA, Bouw MP and Pillay J: $\mathrm{C} 1$-esterase inhibitor attenuates the inflammatory response during human endotoxemia. Crit Care Med 38: 2139-2145, 2010.

7. Pevet P and Challet E: Melatonin: both master clock output and internal time-giver in the circadian clocks network. J Physiol Paris 105: 170-182, 2011.

8. Brzezinski A: Melatonin in humans. N Engl J Med 336: 186-195, 1997.

9. Escames G, Leon J, Macias M, Khaldy H and AcunaCastroviejo D: Melatonin counteracts lipopolysaccharide-induced expression and activity of mitochondrial nitric oxide synthase in rats. FASEB J 17: 932-934, 2003.

10. Lee YD, Kim JY, Lee KH, Kwak YJ and Lee SK: Melatonin attenuates lipopolysaccharide-induced acute lung inflammation in sleep-deprived mice. J Pineal Res 46: 53-57, 2009.

11. Mundigler G, Delle-Karth G, koreny M, et al: Impaired circadian rhythm of melatonin secretion in sedated critically ill patients with severe sepsis. Crit Care Med 30: 536-540, 2002.

12. Bourne RS and Mills GH: Melatonin: possible implications for the postoperative and critically ill patient. Intensive Care Med 32: 371-379, 2006.

13. Reppert SM and Weaver DR: Coordination of circadian timing in mammals. Nature 418: 935-941, 2002.

14. James FO, Cermakian N and Boivin DB: Circadian rhythms of melatonin, cortisol, and clock gene expression during simulated night shift work. Sleep 30: 1427-1436, 2007.

15. O'Connor MF, Motivala SJ, Valladares EM, Olmstead R and Irwin MR: Sex differences in monocyte expression of IL-6: role of autonomic mechanisms. Am J Physiol Regul Integr Comp Physiol 293: R145-R151, 2007.

16. Haimovich B, Calvano J, Haimovich AD, Calvano SE and Coyle SM: In vivo endotoxin synchronizes and suppresses clock gene expression in human peripheral blood leukocytes. Crit Care Med 38: 751-758, 2010.

17. Pollard LC, Choy EH, Gonzalez J, Khoshaba B and Scott DL: Fatigue in rheumatoid arthritis reflects pain, not disease activity. Rheumatology (Oxford) 45: 885-889, 2006.

18. American College of Chest Physicians/Society of Critical Care Medicine consensus conference: Definitions of sepsis and organ failure and guidelines for the use of innovative therapies in sepsis. Crit Care Med 20: 864-874, 1992.

19. Kusanagi H, Hida A and Satoh K: Expression profiles of 10 circadianclock genes in human peripheral blood mononuclear cells. Neurosci Res 61: 136-142, 2008.

20. Verceles AC, Silhan L and Terrin M: Circadian rhythm disruption in severe sepsis: the effect of ambient light on urinary 6-sulfatoxymelatonin secretion. Intensive Care Med 38: 804-810, 2012.

21. Liu J, Malkani G and Shi X: The circadian clock Period 2 gene regulates gamma interferon production of NK cells in host response to lipopolysaccharide-induced endotoxic shock. Infect Immun 74: 4750-4756, 2006.

22. Okada K, Yano M, Doki Y, Azama T and Iwanaga H: Injection of LPS causes transient suppression of biological clock genes in rats. J Surg Res 145: 5-12, 2008.

23. Teboul M, Barrat-Petit MA and Li XM: Atypical patterns of circadian clock gene expression in human peripheral blood mononuclear cells. J Mol Med 83: 693-699, 2005.

24. Godin PJ and Buchman TG: Uncoupling of biological oscillators: A complementary hypothesis concerning the pathogenesis of multiple organ dysfunction syndrome. Crit Care Med 24: 1107-1111, 1996. 
25. Steinhilber D, Brungs M, Werz O, Wiesenberg I and Danielsson C: The nuclear receptor for melatonin repress 5-lipoxygenase gene expression in human B lymphocytes. J Biol Chem 270: 7037-7040, 1995.

26. Deng WG, Tang ST, Tseng HP and Wu KK: Melatonin suppresses cyclooxygenase-2 and inducible nitric oxide synthase expression by inhibiting p52 acetylation and binding. Blood 108: 518-524, 2006.
27. Carrillo-Vico A, Lardone PJ, Naji L, Fernandez-Santos JM and Martin-Lacave I: Beneficial pleiotropic actions of melatonin in an experimental model of septic shock in mice: regulation of proanti-inflammatory cytokine network, protection against oxidative damage and anti-apoptotic effects. J Pineal Res 39: 400-408, 2005.

28. Shang Y, Xu SP, Wu Y, Jinag YX and Wu ZY: Melatonin reduces acute lung injury in endotoxemic rats. Chin Med J(Engl) 122: $1388-1393,2009$ 